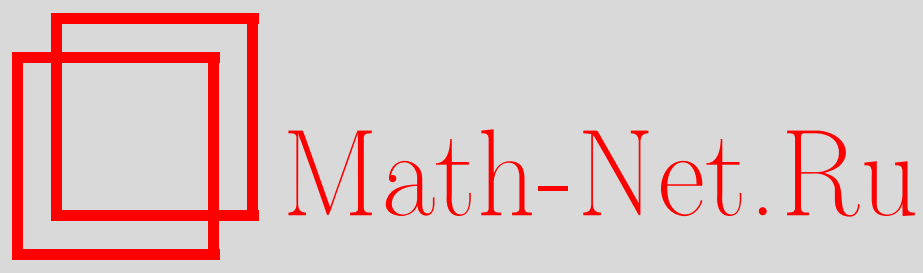

А. Г. Мешков, Нелокальные симметрии двухполевых дивергентных эволюционных систем, ТМФ, 2008, том 156, номер 3, 351-363

DOI: https://doi.org/10.4213/tmf6252

Использование Общероссийского математического портала Math-Net.Ru подразумевает, что вы прочитали и согласны с пользовательским соглашением http://www.mathnet.ru/rus/agreement

Параметры загрузки:

IP : 44.207 .124 .84

26 апреля 2023 г., 16:01:43

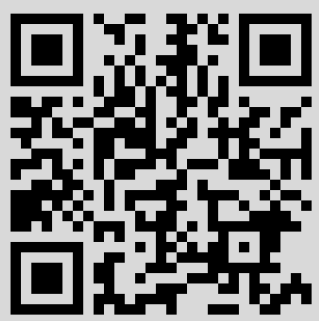




\title{
НЕЛОКАЛЬНЫЕ СИММЕТРИИ ДВУХПОЛЕВЫХ ДИВЕРГЕНТНЫХ ЭВОЛЮЦИОННЫХ СИСТЕМ
}

\begin{abstract}
Вычислены нелокальные симметрии для двухполевых дивергентных точно интегрируемых эволюционных уравнений третьего порядка. Эти симметрии, рассматриваемые как эволюционные уравнения, коммутируют с высшими аналогами уравнений, по которым они строились, и, видимо, являются точно интегрируемыми. Путем дифференцирования нелокальных систем и замен переменных получены локальные гиперболические системы, а также неэволюционные системы третьего порядка. Для некоторых новых систем найдены их представления нулевой кривизны.
\end{abstract}

Ключевые слова: сохраняющаяся плотность, слабо нелокальная переменная, нелокальная симметрия, отрицательный поток, точная интегрируемость.

\section{1. ВВЕДЕНИЕ}

В данной статье мы вычисляем нелокальные симметрии для систем, найденных в [1] путем симметрийной классификации. Эти вычисления не требуют предварительного знания представления Лакса или представления нулевой кривизны. Проведенное исследование позволило найти, кроме известных цепочек Тоды, некоторые новые системы.

Поясним наш подход на примере уравнения sin-Гордон.

В работе [2], по-видимому, впервые были исследованы обобщенные симметрии уравнения sin-Гордон

$$
v_{t x}=\sin v .
$$

Оказалось, что одна из симметрий совпадает с модифицированным уравнением Кортевега-де Фриза (мКдФ)

$$
v_{t}=v_{x x x}+\frac{1}{2} v_{x}^{3}
$$

На этот результат можно взглянуть иначе: записав уравнение sin-Гордон в эволюционном виде $u_{t}=\partial_{x}^{-1} \sin u$, можем сказать, что это нелокальное уравнение является симметрией уравнения мКдФ.

* Орловский государственный технический университет, Орел, Россия. E-mail: a_meshkov@orel.ru 
Рассмотрим это подробнее. Для упрощения формул введем функцию $u=i v_{x}$, которая удовлетворяет следующему уравнению:

$$
u_{t}=u_{x x x}-\frac{3}{2} u^{2} u_{x}
$$

Очевидно, что $u$ - сохраняющаяся плотность, поэтому можно ввести нелокальную переменную $w=D_{x}^{-1} u$ (подробнее см. в разделе 2). Далее, нетрудно проверить, что функции $e^{w}$ и $e^{-w}$ также являются сохраняющимися плотностями уравнения (3). Это позволяет ввести еще две нелокальные переменные $w_{1}=D_{x}^{-1} e^{w}, w_{2}=D_{x}^{-1} e^{-w}$.

Известно (см., например, [3]), что уравнение (3) допускает следующую симметрию:

$$
u_{\tau}=c_{1} e^{w}+c_{2} e^{-w}+c_{3}\left(w_{1} e^{-w}+w_{2} e^{w}\right),
$$

где $c_{i}$ - произвольные постоянные.

Если $c_{3}=0$, то, принимая в (4) $w$ за новую неизвестную функцию и заменяя $u=w_{x}$, получаем гиперболическое уравнение

$$
w_{\tau x}=c_{1} e^{w}+c_{2} e^{-w}
$$

Выбирая конкретные значения величин $c_{1}$ и $c_{2}$, очевидно, можно получить уравне-

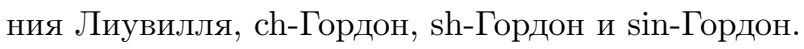

Если продифференцировать уравнение (4) при $c_{3}=0$, получаем $u_{\tau x}=u\left(c_{1} e^{w}-\right.$ $\left.c_{2} e^{-w}\right)$. Исключая $w$ при помощи исходного уравнения, приходим к другому интегрируемому уравнению

$$
u_{\tau x}=u \sqrt{u_{\tau}^{2}-4 c_{1} c_{2}} .
$$

При этом соотношение $u=w_{x}$ связывает (5) и (6).

Если $c_{3} \neq 0$, то, дифференцируя уравнение (4) и комбинируя результат с исходным уравнением, получаем $\left(u^{-1} u_{\tau x}\right)_{x}=u u_{\tau}-2 c_{3} u^{-2} u_{x}$. Растяжением переменной $\tau$ можно изменить $c_{3}$. Полагая $c_{3}=-1 / 2$, окончательно имеем

$$
u_{\tau x x}=u^{-1} u_{x}\left(u_{\tau x}+1\right)+u^{2} u_{\tau} .
$$

Положив здесь $u_{\tau x}=u z_{\tau}$, получаем систему гиперболических уравнений:

$$
z_{\tau x}=u u_{\tau}+u^{-2} u_{x}, \quad u_{\tau x}=u z_{\tau} .
$$

Можно показать, что нелокальное уравнение (4) имеет представление Лакса, поэтому все его следствия, полученные дифференцированием, также имеют представления Лакса. Таким образом, одно интегрируемое эволюционное уравнение (3) порождает множество неэволюционных интегрируемых уравнений (5)-(8).

В литературе известно несколько методов построения новых интегрируемых уравнений по уравнению, имеющему представление Лакса или представление нулевой кривизны. Исключая из рассмотрения высшие аналоги интегрируемого уравнения, можно, видимо, утверждать, что все методы сводятся к заменам зависимых переменных. При этом новые переменные могут быть как локальными, так и нелокальными функциями исходных переменных. Чтобы новое уравнение было неэволюционным, 
замена переменных должна быть, очевидно, нелокальной. Нам известны три способа введения нелокальных переменных.

Во-первых, в качестве новых переменных можно принять коэффициенты асимптотического разложения следа резольвенты оператора Лакса [4]. Если пользоваться современной терминологией, то можно сказать, что эти коэффициенты содержат интегралы от канонических сохраняющихся плотностей $\rho_{n}$. Эти же плотности $\rho_{n}$ еще раньше выводились при помощи асимптотических разложений собственных функций операторов Лакса [5]. Впоследствии было показано (см. [6]), что интегралы $w_{n}=D_{x}^{-1} \rho_{n}$ можно использовать как новые динамические переменные и их можно строить по любым сохраняющимся плотностям, а не только по каноническим.

Во-вторых, в качестве новых переменных можно принять собственные функции оператора Лакса, соответствующие различным собственным значениям [7].

В-третьих, в качестве новых переменных можно принять зависимые переменные, полученные в результате нескольких преобразований Беклунда [8]. В работе [9] предложена некоторая модификация этого метода.

В этой работе мы фактически используем первый способ. Однако новые нелокальные переменные вводятся с помощью классических законов сохранения, которые вычисляются непосредственно.

\section{2. ОСНОВНЫЕ ПОНЯТИЯ И ОБОЗНАЧЕНИЯ}

Рассмотрим эволюционную систему с двумя независимыми переменными $t, x$ и $m$ зависимыми переменными $u^{\alpha}$ :

$$
u_{t}=K\left(t, x, u, u_{x}, \ldots, u_{n}\right)
$$

где $K=\left\{K^{\alpha}, \alpha=1,2, \ldots, m\right\}$ и $u=\left\{u^{\alpha}, \alpha=1,2, \ldots, m\right\}$ - бесконечно дифференцируемые вектор-функции, $u=u_{0}, u_{x}=u_{1}, u_{k}=\partial^{k} u / \partial x^{k}$. Совокупность всех зависимых переменных $u_{i}^{\alpha}$ будем обозначать одной буквой $u$ для сокращения записи.

ОПРЕДЕЛЕНИЕ 1 [10]. Обобщенной симметрией системы (9) называется векторфункция $\sigma\left(t, x, u, u_{x}, \ldots, u_{k}\right)$, удовлетворяющая уравнению

$$
\left(D_{t}-K_{*}\right) \sigma=0
$$

где

$$
\begin{gathered}
\left(K_{*}\right)_{\beta}^{\alpha}=\sum_{k \geqslant 0} \frac{\partial K^{\alpha}}{\partial u_{k}^{\beta}} D_{x}^{k}, \\
D_{x}=\frac{\partial}{\partial x}+\sum_{\alpha, k \geqslant 0} u_{k+1}^{\alpha} \frac{\partial}{\partial u_{k}^{\alpha}}, \quad D_{t}=\frac{\partial}{\partial t}+\sum_{\alpha, k \geqslant 0}\left(D_{x}^{k} K^{\alpha}\right) \frac{\partial}{\partial u_{k}^{\alpha}} .
\end{gathered}
$$

Здесь $D_{x}$ - оператор полного дифференцирования по $x, D_{t}$ - оператор эволюционного дифференцирования.

Порядок дифференциального оператора $f_{*}$ называется порядком вектор-фуункu, u $f$. 
Обобщенные симметрии часто записывают в виде эволюционных систем

$$
u_{\tau}=\sigma(u)
$$

где $\tau$ - новый параметр эволюции. Ясно, что для получения неэволюционных интегрируемых уравнений следует искать симметрии, не зависящие явно от $t$.

ОПРЕДЕЛЕНИЕ 2 [10]. Если на любом решении системы (9) выполнено тождество

$$
D_{t} \rho(t, x, u)=D_{x} \theta(t, x, u),
$$

где $\rho$ и $\theta$ - некоторые дифференцируемые функции, то соотношение (13) называется локальным законом сохранения системы (9). Функция $\rho$ называется сохраняющейся плотностъю, а функция $\theta$ - плотностъю тока. Пару функций $(\rho, \theta)$ называют также сохраняющимся током.

Операторы (11) перестановочны, поэтому $\rho_{0}=D_{x} f$ и $\theta_{0}=D_{x} f-\operatorname{coxраняющийся~}$ ток при произвольной функции $f$. Такие токи называются тривиальными. Сохраняющиеся токи всегда определяют с точностью до тривиальных слагаемых.

Пусть $(\rho, \theta)$ - сохраняющийся ток, тогда система

$$
D_{x} w=\rho(t, x, u), \quad D_{t} w=\theta(t, x, u)
$$

совместна при любой функции $u$, удовлетворяющей уравнению (9). Решение уравнения (14) формально записывается в виде $w=D_{x}^{-1} \rho$. Можно рассматривать $w$ как новую динамическую переменную, она называется слабо нелокальной или квазилокальной [6]. Такие переменные мы будем называть нелокальными переменными первого порядка. Пусть $\left\{\rho_{i}, \theta_{i}\right\}$ - локальные сохраняющиеся токи, а $\left\{w_{i}^{(1)}=D_{x}^{-1} \rho_{i}\right\}-$ соответствующие нелокальные переменные первого порядка. Если существуют сохраняющиеся токи, зависящие от $w_{i}^{(1)}$ и, возможно, от локальных переменных, то по ним можно определить новые слабо нелокальные переменные и т.д.

Порядок нелокальных переменных определим индуктивно. Пусть определены переменные $w^{(1)}, \ldots, w^{(n)}$ до $n$-го порядка. Тогда, если имеется нетривиальная сохраняющаяся плотность $\rho\left(t, x, u, w^{(1)}, \ldots, w^{(n)}\right)$ и переменные $w_{i}^{(n)}$ не устраняются калибровочным преобразованием $\rho \rightarrow \rho+D_{x} f, \theta \rightarrow \theta+D_{t} f$, то переменную $w=D_{x}^{-1} \rho\left(t, x, u, w^{(1)}, \ldots, w^{(n)}\right)$ назовем нелокальной переменной $(n+1)$-го порядка.

Операторы (11) продолжаются на нелокальные переменные $w_{i}$ всех порядков по формулам

$$
\widehat{D}_{x}=D_{x}+\rho_{i} \frac{\partial}{\partial w_{i}}, \quad \widehat{D}_{t}=D_{t}+\theta_{i} \frac{\partial}{\partial w_{i}},
$$

где по $i$ подразумевается суммирование, $\left(\rho_{i}, \theta_{i}\right)$ - сохраняющиеся токи, по которым определяются переменные $w_{i}$ с помощью (14). Известно, что продолженные операторы $\widehat{D}_{x}$ и $\widehat{D}_{t}$ коммутируют (см., например, [11]). Это означает, в частности, что определяющее уравнение для нелокальных симметрий получается из (10) заменой операторов (11) на (15). Если уравнение (10) с продолженным оператором имеет решение, зависящее от нелокальных переменных, то это решение называется нелокальной симметрией. 
Для приложений наиболее интересны дифференциальные уравнения невысокого порядка. Поэтому для получения интересных интегрируемых гиперболических систем мы рассматриваем сохраняющиеся плотности и нелокальные симметрии, включающие только переменные $u^{\alpha}$ и $u_{1}^{\alpha}$. Нелокальные переменные порядка больше двух не вычислялись, потому что зависящие от этих переменных симметрии чрезвычайно громоздкие.

Если система уравнений имеет вид

$$
u_{t}^{\alpha}=D_{x} K^{\alpha}\left(t, x, u, u_{x}, \ldots, u_{n-1}\right),
$$

то ее называют дивергентной. Полагая в $(16) u^{\alpha}=U_{x}^{\alpha}$, получаем систему

$$
U_{t}^{\alpha}=K^{\alpha}\left(t, x, U_{x}, \ldots, U_{n}\right)
$$

обычно называемую потенциальной версией системы (16), так как $U$ является потенциалом для $u$.

Далее мы рассматриваем системы вида (17) с двумя независимыми функциями $u$ и $v$, поэтому в общих формулах, рассмотренных выше, следует заменять $u^{1}$ и $u^{2}$ на $u$ и $v$ соответственно.

\section{3. НЕЛОКАЛЬНЫЕ СИММЕТРИИ}

Некоторые из систем, представленных в работе [1], не имеют нелокальных симметрий рассматриваемого типа, а другие допускают многопараметрические нелокальные симметрии. Произвольные постоянные, входящие в симметрии, обозначаются как $c_{i}$ или $k_{i}$.

3.1. Первая из систем

$$
u_{t}=u_{3}+\frac{3}{2} u_{1} v_{2}-\frac{3}{4} u_{1} v_{1}^{2}+\frac{1}{4} u_{1}^{3}, \quad v_{t}=-\frac{1}{2} v_{3}-\frac{3}{4}\left(2 u_{1} u_{2}+u_{1}^{2} v_{1}\right)+\frac{1}{4} v_{1}^{3}
$$

найдена ранее в [12] и связана с аффинной алгеброй $B_{2}^{(1)}$. Она допускает следующую нелокальную симметрию:

$$
\begin{aligned}
u_{\tau}=c_{2} & w_{2}+c_{3} w_{3}+c_{4}\left(w_{4}-w_{1} w_{2}\right)+c_{5}\left(w_{5}-w_{1} w_{3}\right)+ \\
& +c_{6}\left(w_{3} w_{4}-w_{2} w_{5}\right)+c_{7}\left(2 w_{7}-w_{3} w_{1}^{2}\right)+ \\
& +c_{8}\left(w_{2} w_{7}+w_{3} w_{6}+w_{4} w_{5}-w_{1} w_{3} w_{4}-w_{1} w_{2} w_{5}\right)+ \\
& +c_{9}\left(w_{1} w_{3} w_{4}+w_{1} w_{2} w_{5}-2 w_{3} w_{6}-w_{4} w_{5}\right), \\
v_{\tau}=c_{1} & w_{1}-c_{2} w_{2}+c_{3} w_{3}+c_{4} w_{1} w_{2}-c_{5} w_{1} w_{3}+ \\
& +c_{6}\left(w_{3} w_{4}+w_{2} w_{5}-w_{8}\right)-c_{7}\left(2 w_{7}-2 w_{1} w_{5}+w_{3} w_{1}^{2}\right)+ \\
& +c_{8}\left(w_{3} w_{6}-w_{2} w_{7}-w_{1} w_{3} w_{4}+w_{1} w_{2} w_{5}\right)+ \\
& +c_{9}\left(w_{3} w_{1} w_{4}+w_{1} w_{8}-2 w_{3} w_{6}-w_{1} w_{2} w_{5}\right),
\end{aligned}
$$

где

$w_{1}=D_{x}^{-1} e^{v}, \quad w_{2}=D_{x}^{-1} e^{u-v}, \quad w_{3}=D_{x}^{-1} e^{-u-v}, \quad w_{4}=D_{x}^{-1} w_{2} e^{v}, \quad w_{5}=D_{x}^{-1} w_{3} e^{v}$, 


$$
w_{6}=D_{x}^{-1} w_{1} w_{2} e^{v}, \quad w_{7}=D_{x}^{-1} w_{1} w_{3} e^{v}, \quad w_{8}=D_{x}^{-1} w_{2} w_{3} e^{v} .
$$

Мы проверили, что поток (19) коммутирует не только с потоком (18), но и со следующим элементом из иерархии интегрируемых систем:

$$
\begin{aligned}
u_{t}=u_{5} & +\frac{5}{4} v_{4} u_{1}+\frac{5}{4} u_{3}\left(2 v_{2}-v_{1}^{2}\right)+\frac{5}{4} v_{3}\left(2 u_{2}-u_{1} v_{1}\right)-\frac{5}{2} u_{2} v_{2} v_{1}-\frac{5}{8} v_{2}^{2} u_{1}- \\
& \quad-\frac{5}{8} u_{1} v_{2}\left(u_{1}^{2}+v_{1}^{2}\right)+\frac{1}{32} u_{1}\left(5 v_{1}^{5}-3 u_{1}^{4}+10 u_{1}^{2} v_{1}^{2}\right), \\
v_{t}=- & \frac{1}{4} v_{5}-\frac{5}{4} u_{1} u_{4}-\frac{5}{4} u_{3}\left(u_{2}+u_{1} v_{1}\right)-\frac{5}{8} v_{3}\left(u_{1}^{2}-v_{1}^{2}\right)+\frac{5}{8} v_{1} v_{2}^{2}-\frac{5}{4} u_{1} u_{2} v_{2}+ \\
& +\frac{5}{8} u_{1} u_{2}\left(u_{1}^{2}+v_{1}^{2}\right)+\frac{1}{32} v_{1}\left(5 u_{1}^{4}-3 v_{1}^{4}+10 u_{1}^{2} v_{1}^{2}\right) .
\end{aligned}
$$

Поэтому есть основания надеяться на точную интегрируемость системы (19).

Для всех рассматриваемых ниже систем мы также проверили коммутативность нелокальных симметрий с высшими элементами из соответствующих иерархий, и далее мы об этом не упоминаем. Высшие элементы иерархий не приводятся для уменьшения объема статьи.

3.1.1. Положив в (19) $c_{i}=0, i>3$, получаем цепочку Тоды:

$$
u_{\tau x}=c_{2} e^{u-v}+c_{3} e^{-u-v}, \quad v_{\tau x}=c_{1} e^{v}-c_{2} e^{u-v}+c_{3} e^{-u-v} .
$$

Согласно обозначениям работы [12] запишем систему $u_{i, t x}=\exp \left[\sum_{j} u_{j} A_{j i}\right]$, где $A_{j i}$, $i, j=1,2,3,-$ матрица Картана аффинной алгебры $D_{3}^{(2)}$ с диаграммой Дынкина $\circ \Longleftarrow$ о о. В явном виде имеем

$$
u_{1, t x}=\exp \left[2 u_{1}-2 u_{3}\right], \quad u_{2, t x}=\exp \left[2 u_{2}-2 u_{3}\right], \quad u_{3, t x}=\exp \left[2 u_{3}-u_{1}-u_{2}\right] .
$$

Легко видеть, что функции $p=u_{1}-u_{3}, q=u_{2}-u_{3}$ удовлетворяют системе $p_{t x}=$ $e^{2 p}-e^{-p-q}, q_{t x}=e^{2 q}-e^{-p-q}$. Эта же система получается, если в системе $(20)$ выполнить подстановку $u-v=2 p, u+v=-2 q$ и избавиться от постоянных $c_{i} \neq 0$ сдвигами по $p, q, x$. Однако постоянные в (20) могут быть и нулевыми. В частности, в случае $c_{1}=0$ система распадается на два уравнения Лиувилля.

Систему (20) можно представить в различных формах, выбирая в качестве неизвестных функций нелокальные переменные. Например, при $p=w_{1}, q=w_{2}$ получаem

$$
p_{\tau x}=p_{x}\left(c_{1} p-c_{2} q+c_{3} w\right), \quad q_{\tau x}=q_{x}\left(2 c_{2} q-c_{1} p\right), \quad w_{x}=p_{x}^{-2} q_{x}^{-1} .
$$

3.1.2. Если в (19) $c_{4}=1$ и $c_{i}=0, i>4$, то имеется несколько возможностей.

$A$. Пусть $p=w_{1}$ и $q=\ln w_{2}$ - новые неизвестные функции. Тогда при $c_{1}=c_{3}=0$ получаем

$$
p_{\tau x}=e^{q} p p_{x}, \quad q_{\tau x}=-e^{q} p q_{x}+f(\tau) q_{x},
$$

где $f(\tau)$ - "постоянная" интегрирования.

Б. Пусть $c_{3}=0$, тогда подстановка

$$
u=\ln \left(U_{x}\left(\frac{V_{x}}{U_{x}}\right)_{x}\right), \quad v=\ln U_{x}, \quad w_{1}=U, \quad w_{2}=\frac{V_{x}}{U_{x}}, \quad w_{4}=V
$$


приводит к уравнениям, отличным от (21):

$$
U_{\tau x}=c_{1} U U_{x}-c_{2} V_{x}+U V_{x}, \quad V_{\tau x}=c_{1} V U_{x}+V V_{x}+f(\tau) U_{x}
$$

Отметим, что если в (19) $c_{4}=0, c_{5} \neq 0$, то, немного изменив вид предыдущей подстановки, снова придем к системе (22). Если при выводе системы (22) считать, что $c_{3} \neq 0$, то возникают более сложные уравнения третьего порядка.

$B$. Дифференцируя дважды уравнения (19) при произвольных $c_{1}, c_{2}, c_{3}$, получаем локальную систему третьего порядка

$$
u_{\tau x x}=\left(2 u_{x}+z_{x}\right) u_{\tau x}+c u_{x} e^{z}-e^{u}, \quad z_{\tau x x}=-\left(z_{x}+u_{x}\right) z_{\tau x}+c\left(u_{x}+2 z_{x}\right) e^{z}-e^{u},
$$

где $z=-u-v, c=-2 c_{3}$.

3.1.3. Положим в (19) $c_{7}=-1, c_{i}=0, i>3$. Тогда эта система приводится к следующему виду:

$$
\begin{aligned}
& u_{t x x}=-u_{t x}\left(p_{x}+2 u_{x}\right)+2 c_{2} u_{x} e^{-p}+2 \sqrt{u_{t x} e^{p}-c_{2}-c_{3} e^{-2 u}} \\
& p_{t x x}=p_{t x}\left(u_{x}+p_{x}\right)+2 c_{2}\left(u_{x}+2 p_{x}\right) e^{-p}+2 \sqrt{u_{t x} e^{p}-c_{2}-c_{3} e^{-2 u}}
\end{aligned}
$$

где $p=v-u$.

3.2. Вторая интегрируемая система

$$
\begin{aligned}
& u_{t}=u_{3}-3 v_{3}+3 v_{2}\left(v_{1}-2 u_{1}\right)+3 u_{1} v_{1}^{2}-2 u_{1}^{3}, \\
& v_{t}=-3 u_{3}+4 v_{3}-3 u_{2}\left(v_{1}-2 u_{1}\right)+3 v_{1} u_{1}^{2}-2 v_{1}^{3},
\end{aligned}
$$

найдена ранее в [12] и связана с аффинной алгеброй $A_{4}^{(2)}$. Она допускает следующую нелокальную симметрию:

$$
\begin{gathered}
u_{\tau}=c_{2} w_{2}+c_{3} w_{3}+c_{4} w_{4}+c_{5}\left(2 w_{2} w_{3}-w_{5}\right)+c_{6} w_{6}+ \\
+c_{7}\left(2 w_{3} w_{4}-w_{7}\right)+c_{8}\left(2 w_{3} w_{6}-w_{9}\right)+c_{9} w_{2}\left(w_{2} w_{3}-w_{5}\right)- \\
-c_{10}\left(w_{5} w_{6}+w_{2} w_{9}-2 w_{4} w_{7}+2 w_{3} w_{4}^{2}-2 w_{2} w_{3} w_{6}\right), \\
v_{\tau}=c_{1} w_{1}+c_{2} w_{2}+c_{4} w_{1} w_{2}+c_{5} w_{5}+c_{6}\left(2 w_{1} w_{4}-w_{6}\right)+ \\
+c_{7} w_{1} w_{5}+c_{8}\left(2 w_{1} w_{7}-w_{9}\right)+c_{9}\left(2 w_{8}-w_{2} w_{5}\right)+ \\
+c_{10}\left(w_{5} w_{6}-w_{2} w_{9}-2 w_{1} w_{4} w_{5}+2 w_{1} w_{2} w_{7}\right)
\end{gathered}
$$

где

$$
\begin{array}{lll}
w_{1}=D_{x}^{-1} e^{v}, & w_{2}=D_{x}^{-1} e^{-u-v}, & w_{3}=D_{x}^{-1} e^{2 u}, \\
w_{4}=D_{x}^{-1} w_{1} e^{-u-v}, & w_{5}=D_{x}^{-1} w_{3} e^{-u-v}, & w_{6}=D_{x}^{-1} w_{1}^{2} e^{-u-v} \\
w_{7}=D_{x}^{-1} w_{1} w_{3} e^{-u-v}, & w_{8}=D_{x}^{-1} w_{2} w_{3} e^{-u-v}, & w_{9}=D_{x}^{-1} w_{1}^{2} w_{3} e^{-u-v} .
\end{array}
$$

Приведем простые локальные системы, вытекающие из (25). 
3.2.1. Положив $c_{i}=0$ при $i>3$, получаем цепочку Тоды:

$$
u_{\tau x}=c_{2} e^{-u-v}+c_{3} e^{2 u}, \quad v_{\tau x}=c_{1} e^{v}+c_{2} e^{-u-v} .
$$

Если записать систему $u_{i, t x}=\exp \left[\sum_{j} u_{j} A_{j i}\right]$, где $A_{j i}-$ матрица Картана аффинной алгебры $A_{4}^{(2)}$ с диаграммой Дынкина $\circ \Longrightarrow \circ \Longrightarrow$, и положить $u=2 u_{1}-u_{2}, v=$ $2 u_{3}-u_{2}$, то получим систему (26) с постоянными $c_{1}=2, c_{2}=-1, c_{3}=1$. Систему (26) можно записать в различных формах. Например, при $p=w_{1}, q=w_{2}$ имеем

$$
p_{\tau x}=p_{x}\left(c_{1} p+c_{2} q\right), \quad q_{\tau x}=-q_{x}\left(c_{1} p+2 c_{2} q+c_{3} w\right), \quad w_{x}=\left(p_{x} q_{x}\right)^{-2} .
$$

3.2.2. Если $c_{i}=0, i>4, c_{4}=1$, то двукратное дифференцирование уравнений (25) приводит к следующей системе:

$$
\begin{aligned}
& u_{\tau x x}=-u_{\tau x}\left(u_{x}+v_{x}\right)+c\left(3 u_{x}+q_{x}\right) e^{2 u}+e^{-u}, \quad c=c_{3}, \\
& v_{\tau x x}=v_{x} v_{\tau x}-u_{\tau x}\left(u_{x}+2 v_{x}\right)+c\left(u_{x}+2 v_{x}\right) e^{2 u}+2 e^{-u} .
\end{aligned}
$$

Очевидно, что порядок второго уравнения можно понизить подстановкой $v_{x} \rightarrow v$. Если принять, при прежних условиях, $p=w_{1}$ и $q=w_{2}$ за новые функции, то получаем систему следующего вида:

$$
p_{\tau x}=p_{x}\left(c_{1} p+c_{2} q+p q\right), \quad\left(\ln q_{x}\right)_{\tau x}=p_{x}\left(c_{1}+q\right)-c_{3} p_{x}^{-2} q_{x}^{-2} .
$$

3.2.3. Если $c_{4}=0, c_{5}=1, c_{i}=0, i>5$, то двукратное дифференцирование уравнений (25) приводит к следующей системе:

$$
\begin{aligned}
& u_{\tau x x}=2 u_{x} u_{\tau x}-v_{\tau x}\left(3 u_{x}+v_{x}\right)+c\left(3 u_{x}+v_{x}\right) e^{v}+3 e^{u-v}, \\
& v_{\tau x x}=-v_{\tau x}\left(u_{x}+v_{x}\right)+c\left(u_{x}+2 v_{x}\right) e^{v}+e^{u-v}, \quad c=c_{1} .
\end{aligned}
$$

3.2.4. Если $c_{6}=1, c_{i}=0, i>4$, то приходим к следующей системе:

$$
\begin{aligned}
& u_{\tau x x}=-2 u_{\tau x}\left(u_{x}+q_{x}\right)+2 c\left(2 u_{x}+q_{x}\right) e^{2 u}+2 \sqrt{u_{\tau x} e^{2 q}+b e^{-2 u}-c e^{2(u+q)}} \\
& q_{\tau x x}=q_{\tau x}\left(u_{x}+2 q_{x}\right)+\frac{1}{2} c\left(2 q_{x}-u_{x}\right) e^{2 u}+\sqrt{u_{\tau x} e^{2 q}+b e^{-2 u}-c e^{2(u+q)}}
\end{aligned}
$$

где $b=c_{4}^{2} / 4-c_{2}, c=c_{3}, q=(v-u) / 2$. Заметим, что в случае $b=c=0$ порядок первого уравнения можно понизить подстановкой $u_{x} \rightarrow u$.

3.3. Система

$$
u_{t}=u_{3}+u_{1} v_{2}-u_{1} v_{1}^{2}, \quad v_{t}=u_{2} u_{1}+u_{1}^{2} v_{1}
$$

найдена ранее в [12] и связана с аффинной алгеброй $A_{3}^{(2)}$. Она допускает следующую нелокальную симметрию:

$$
\begin{gathered}
u_{\tau}=c_{1} w_{1}+c_{2} w_{2}+c_{4}\left(w_{4}-w_{1} w_{3}\right)+c_{5}\left(w_{5}-w_{1} w_{4}\right)+ \\
+c_{6}\left(w_{6}-w_{2} w_{3}\right)+c_{7}\left(w_{1} w_{6}-w_{2} w_{4}\right)+c_{8}\left(w_{8}-w_{2} w_{6}\right), \\
v_{\tau}=-c_{1} w_{1}+c_{2} w_{2}+c_{3} w_{3}+c_{4}\left(w_{4}+w_{1} w_{3}\right)+c_{5} w_{1} w_{4}- \\
-c_{6}\left(w_{6}+w_{2} w_{3}\right)-c_{7}\left(w_{1} w_{6}+w_{2} w_{4}\right)-c_{8} w_{2} w_{6}
\end{gathered}
$$


где

$$
\begin{array}{llll}
w_{1}=D_{x}^{-1} e^{u-v}, & w_{2}=D_{x}^{-1} e^{-u-v}, & w_{3}=D_{x}^{-1} e^{2 v}, & w_{4}=D_{x}^{-1} w_{1} e^{2 v}, \\
w_{5}=D_{x}^{-1} w_{1}^{2} e^{2 v}, & w_{6}=D_{x}^{-1} w_{2} e^{2 v}, & w_{7}=D_{x}^{-1} w_{1} w_{2} e^{2 v}, & w_{8}=D_{x}^{-1} w_{2}^{2} e^{2 v} .
\end{array}
$$

3.3.1. В случае $c_{i}=0, i>3$, получаем следующую цепочку Тоды:

$$
u_{\tau x}=c_{1} e^{u-v}+c_{2} e^{-u-v}, \quad v_{\tau x}=-c_{1} e^{u-v}+c_{2} e^{-u-v}+c_{3} e^{2 v} .
$$

При $p=u-v, q=-u-v$ эта система имеет вид

$$
p_{\tau x}=2 c_{1} e^{p}-c_{3} e^{-p-q}, \quad q_{\tau x}=-2 c_{2} e^{q}-c_{3} e^{-p-q} .
$$

Отсюда легко найти, что система $(32)$ связана с аффинной алгеброй $C_{2}^{(1)}$, имеющей диаграмму Дынкина $\circ \Longrightarrow 0 \Longleftarrow$. Видно, что в случае $c_{3}=0$ эта система распадается на независимые уравнения Лиувилля.

3.3.2. Если $c_{4} \neq 0, c_{i}=0, i>4$, то при $p=w_{1}, q=w_{3}$ система (31) принимает следующий вид:

$p_{\tau x}=p_{x}\left(2 c_{1} p-c_{3} q-2 c_{4} p q\right), \quad\left(q_{x}^{-1} q_{\tau x}\right)_{x}=2 c_{3} q_{x}-2 c_{1} p_{x}+4 c_{4} p q_{x}+2 c_{4} q p_{x}+2 c_{2} p_{x}^{-1} q_{x}^{-1}$.

Если же просто продифференцировать систему (31), то получим

$u_{\tau x x}=u_{\tau x}\left(2 u_{x}-p_{x}\right)-2 c_{2} u_{x} e^{-p}-c_{4} e^{p}, \quad p_{\tau x x}=2 p_{\tau x}\left(p_{x}-u_{x}\right)+2 c_{2} e^{-p}\left(2 u_{x}-3 p_{x}\right)+2 c_{4} e^{p}$,

где $p=u+v$. Очевидно, что заменой $u_{x} \rightarrow u$ можно понизить порядок первого уравнения.

3.3.3. Если $c_{5} \neq 0$, а остальные $c_{i}=0, i>3$, то дифференцирование системы (31) приводит к следующей системе:

$$
\begin{aligned}
& u_{\tau x x}=u_{\tau x}\left(2 u_{x}+q_{x}\right)-2 c_{2} u_{x} e^{q}+\sqrt{a q_{\tau x} e^{2 u}+b e^{-2 q}+2 a c_{2} e^{2 u+q}} \\
& q_{\tau x x}=-2 q_{\tau x}\left(u_{x}+q_{x}\right)-2 c_{2}\left(2 u_{x}+3 q_{x}\right) e^{q}+2 \sqrt{a q_{\tau x} e^{2 u}+b e^{-2 q}+2 a c_{2} e^{2 u+q}}
\end{aligned}
$$

где $a=-c_{5}, b=-c_{3} c_{5}, q=-u-v$.

Заметим, что системы (29) и (33) совпадают при $c_{2}=c_{3}=c_{4}=0$. Это удивительно, поскольку их симметрии (24) и (30) совершенно разные. В качестве объяснения этого совпадения выскажем следующую гипотезу. Система

$$
u_{\tau x x}=u_{\tau x}\left(2 u_{x}+q_{x}\right)+e^{u} \sqrt{q_{\tau x}}, \quad q_{\tau x x}=-2 q_{\tau x}\left(u_{x}+q_{x}\right)+2 e^{u} \sqrt{q_{\tau x}}
$$

лиувиллевская и обладает двумя последовательностями симметрий, которые строятся по разным интегралам. 
3.4. Система

$$
u_{t}=u_{3}+v_{1} v_{2}-\frac{1}{2} u_{1}^{3}+\frac{1}{2} u_{1} v_{1}^{2}+c_{1} v_{1}, \quad v_{t}=u_{2} v_{1}-\frac{1}{2} u_{1}^{2} v_{1}+\frac{1}{2} v_{1}^{3}-c_{1} u_{1}+c_{2} v_{1}
$$

содержит две существенные константы, от которых зависят и количество, и вид допускаемых симметрий. Это становится понятным, если учесть, что систему (34) можно получить из системы Ито дифференциальной подстановкой, зависящей от $c_{1}$ и $c_{2}$ (см. [1]). Если $c_{1}=0$, то дифференциальная подстановка существенно упрощается, а в случае $c_{1}=c_{2}=0$ подстановка исчезает.

Симметрии системы (34) имеют простой вид только при $c_{1}=0, c_{2} \neq 0$ :

$$
\begin{aligned}
& u_{\tau}=k_{1} w_{1}+k_{2} w_{2}+k_{3}\left(w_{5}+2 w_{2} w_{3}\right)+k_{4}\left(w_{1} w_{5}-4 w_{1} w_{2}+2 w_{2} w_{4}\right) \\
& v_{\tau}=-2 k_{2} e^{-u} v_{x}-4 k_{3} e^{-u} v_{x}\left(2+w_{3}\right)-4 k_{4} e^{-u} v_{x} w_{4}
\end{aligned}
$$

где

$$
\begin{gathered}
w_{1}=D_{x}^{-1} e^{u}, \quad w_{2}=D_{x}^{-1} e^{-u}\left(v_{x}^{2}+c_{2}\right), \quad w_{3}=D_{x}^{-1} e^{u} w_{2}, \\
w_{4}=D_{x}^{-1} e^{u} w_{2} w_{1}, \quad w_{5}=D_{x}^{-1}\left(4 v_{x}^{2} e^{-u}-e^{u} w_{2}^{2}\right) .
\end{gathered}
$$

Если $k_{3}=k_{4}=0$, то дифференцированием системы (35) получаем простую локальную систему

$$
u_{\tau x}=k_{1} e^{u}+k_{2}\left(v_{x}^{2}+c_{2}\right) e^{-u}, \quad v_{\tau}=-2 k_{2} v_{x} e^{-u}
$$

Симметрии системы (34) представлены более полно в расширенном варианте статьи [13]. Все остальные точно интегрируемые системы, найденные в работе [1], не допускают нелокальных симметрий или допускают тривиальные симметрии, приводящие к уравнению Лиувилля.

\section{4. ПРЕДСТАВЛЕНИЯ НУЛЕВОЙ КРИВИЗНЫ}

Здесь мы приводим матрицы $U$ и $V$, входящие в представления нулевой кривизны

$$
U_{\tau}-V_{x}+[U, V]=0
$$

для некоторых из систем, связанных с (24). Спектральный параметр во всех случаях обозначается через $k$.

Система (24) получается из более простой системы Дринфельда-Соколова [12]

$$
\begin{aligned}
m_{t} & =m_{3}-3 n_{3}-3 m_{x}(4 m-9 n)+3 n_{x}(8 m-15 n), \\
n_{t} & =-3 m_{3}+4 n_{3}+12 m_{x} n+6 n_{x}(m-4 n)
\end{aligned}
$$

при помощи следующей дифференциальной подстановки:

$$
m=u_{x}^{2}+\frac{1}{2} v_{x}^{2}-u_{2}-v_{2}, \quad n=u_{x}^{2}-u_{2} .
$$


Поэтому приведем вначале матрицы $U_{0}$ и $V_{0}$, дающие представление нулевой кривизны для системы (36):

$$
U_{0}=\left(\begin{array}{ccccc}
0 & 1 & n-m & 0 & 0 \\
0 & 0 & 0 & 1 & 0 \\
-1 & 0 & 0 & 0 & m-n \\
0 & n & 0 & 0 & k \\
0 & 0 & 1 & 0 & 0
\end{array}\right), \quad V_{0}=\left(\begin{array}{ccccc}
h_{1, x} & h_{2} & f_{1} & 0 & -5 k \\
0 & h_{3, x} & -5 k & -2 h_{3} & 0 \\
-h_{1} & 0 & 0 & 5 & -f_{1} \\
5 k & f_{2} & 0 & -h_{3, x} & k h_{2} \\
0 & 5 & h_{1} & 0 & -h_{1, x}
\end{array}\right),
$$

где

$$
\begin{gathered}
h_{1}=7 n-4 m, \quad h_{2}=m-3 n, \quad h_{3}=4 n-3 m, \\
f_{1}=-4 m_{2}+7 n_{2}+4 m^{2}+7 n^{2}-11 m n, \quad f_{2}=-3 m_{2}+4 n_{2}-8 n^{2}+6 m n .
\end{gathered}
$$

Матрицы (38) принадлежат алгебре $\operatorname{sl}(5, \mathbb{C})$.

Выполнив подстановку (37) в матрицах (38) и устранив $u_{2}$ и $v_{2}$ из матрицы $U_{0}$ калибровочным преобразованием $U=S^{-1}\left(U_{0} S-S_{x}\right), V=S^{-1}\left(V_{0} S-S_{t}\right)$, получаем представление нулевой кривизны для системы (24):

$$
U=\left(\begin{array}{ccccc}
v_{x} & 1 & 0 & 0 & 0 \\
0 & -u_{x} & 0 & 1 & 0 \\
-1 & 0 & 0 & 0 & 0 \\
0 & 0 & 0 & u_{x} & k \\
0 & 0 & 1 & 0 & -v_{x}
\end{array}\right), \quad V=\left(\begin{array}{ccccc}
\varphi_{1} & \varphi_{2} & 0 & -5 v_{x} & -5 k \\
0 & \varphi_{3} & -5 k & \varphi_{4} & 5 k v_{x} \\
-\varphi_{5} & 5 h & 0 & 5 & 0 \\
5 k & 0 & 5 k h & -\varphi_{3} & k \varphi_{2} \\
0 & 5 & \varphi_{5} & 0 & -\varphi_{1}
\end{array}\right),
$$

где

$$
\begin{aligned}
& \varphi_{1}=4 v_{3}-3 u_{3}+3 u_{2}\left(2 u_{x}-v_{x}\right)+3 v_{x} u_{x}^{2}-2 v_{x}^{3} \\
& \varphi_{2}=2 u_{2}-v_{2}-2 u_{x}^{2}-2 v_{x}^{2}+5 u_{x} v_{x} \\
& \varphi_{3}=3 v_{3}-u_{3}+3 v_{2}\left(2 u_{x}-v_{x}\right)-3 u_{x} v_{x}^{2}+2 u_{x}^{3}, \quad h=v_{x}-u_{x}, \\
& \varphi_{4}=2 u_{2}-6 v_{2}-2 u_{x}^{2}+3 v_{x}^{2}, \quad \varphi_{5}=4 v_{2}-3 u_{2}+3 u_{x}^{2}-2 v_{x}^{2} .
\end{aligned}
$$

Из системы $\Psi_{x}=U \Psi$, где матрица $U$ имеет вид (39), нетрудно получить скалярное уравнение

$$
\left(\partial_{x}-u_{x}\right)\left(\partial_{x}+u_{x}\right)\left(\partial_{x}-v_{x}\right) \partial_{x}\left(\partial_{x}-v_{x}\right) \Psi_{5}+k \Psi_{5}=0
$$

из которого ясно, что мы имеем нетривиальное представление нулевой кривизны.

Матрица $U$ для цепочки Тоды (26) указана в (39), а матрица $V$ записывается в следующем виде:

$$
V=\left(\begin{array}{ccccc}
0 & 0 & -c_{1} e^{v} & 0 & 0 \\
-c_{2} e^{-u-v} & 0 & 0 & 0 & 0 \\
0 & 0 & 0 & 0 & c_{1} e^{v} \\
0 & c_{3} e^{2 u} & 0 & 0 & 0 \\
0 & 0 & 0 & -k^{-1} c_{2} e^{-u-v} & 0
\end{array}\right)
$$


Мы предположили, что системы (27)-(29) принадлежат иерархии интегрируемых уравнений, порождаемой системой (24). В таком случае представления нулевой кривизны для всех этих систем должны иметь общую матрицу $U$ (см. (39)). Вычисления подтвердили это предположение, поэтому мы приводим ниже только матрицы $V$ для указанных систем.

Для систем (27), (28), (29) имеем соответственно

$$
\begin{aligned}
& V=\left(\begin{array}{ccccc}
0 & 0 & u_{\tau x}-v_{\tau x}-c e^{2 u} & 0 & 0 \\
c e^{2 u}-u_{\tau x} & 0 & -e^{-u} & 0 & 0 \\
0 & 0 & 0 & -k^{-1} e^{-u} & v_{\tau x}-u_{\tau x}+c e^{2 u} \\
0 & c e^{2 u} & 0 & 0 & 0 \\
0 & 0 & 0 & k^{-1}\left(c e^{2 u}-u_{\tau x}\right) & 0
\end{array}\right) \\
& V=\left(\begin{array}{ccccc}
0 & 0 & -c e^{v} & 0 & 0 \\
c e^{v}-v_{\tau x} & 0 & 0 & 0 & 0 \\
0 & 0 & 0 & 0 & c e^{v} \\
-e^{u-v} & u_{\tau x}-v_{\tau x}+c e^{v} & 0 & 0 & 0 \\
0 & k^{-1} e^{u-v} & 0 & k^{-1}\left(c e^{v}-v_{\tau x}\right) & 0
\end{array}\right), \\
& V=\left(\begin{array}{ccccc}
0 & 0 & -2 q_{\tau x}-c e^{2 u} & 2 k^{-1} e^{2 q} & 0 \\
c e^{2 u}-u_{\tau x} & 0 & -2 r & 0 & 2 e^{2 q} \\
0 & 0 & 0 & -2 k^{-1} r & 2 q_{\tau x}+c e^{2 u} \\
0 & c e^{2 u} & 0 & 0 & 0 \\
0 & 0 & 0 & k^{-1}\left(c e^{2 u}-u_{\tau x}\right) & 0
\end{array}\right)
\end{aligned}
$$

где $r=\sqrt{u_{\tau x} e^{2 q}+b e^{-2 u}-c e^{2(u+q)}}$, а в матрице $U$ для системы (29) (см. (39)) следует выполнить подстановку $v=u+2 q$.

\section{5. ЗАКЛЮЧЕНИЕ}

Как уже отмечалось выше, каждая представленная нелокальная симметрия является симметрией и для указанной эволюционной системы, и для ее высшего аналога. Это дает веские основания надеяться на точную интегрируемость полученных гиперболических систем и систем с высшими производными. Но утверждение о точной интегрируемости какой-либо системы, конечно, нуждается в доказательстве, например путем построения представления нулевой кривизны. Для систем (27)-(29) мы представили такое доказательство, а для других систем эта задача может составить предмет будущего исследования.

Благодарности. Работа выполнена при поддержке РФФИ (грант № 05-0100775-a).

\section{Список литературы}

[1] А. Г. Мешков, Фундамент. и прикл. матем., 12:7 (2006), 141-161.

[2] S. Kumei, J. Math. Phys., 16:12 (1975), 2461-2468. 
[3] Zhijun Qiao, Negative order MKdV hierarchy and a new integrable Neumann-like system, arXiv: nlin/0201065.

[4] И. М. Гельфанд, Л. А. Дикий, УМН, 30:5 (1975), 67-100; Функи. анализ и его прил., 11:2 (1977), 11-27.

[5] В. Е. Захаров, А. Б. Шабат, ЖКЭТФ, 61:1 (1971), 118-134.

[6] И.Ш. Ахатов, Р. К. Газизов, Н. Х. Ибрагимов, "Нелокальные симметрии. Эвристический подход", Итоги науки и техники. Современные проблемы математики. Новейшие достижения, 34, ред. Р. В. Гамкрелидзе, ВИНИТИ, М., 1989, 3-83; В. В. Соколов, С. И. Свинолупов, Матем. заметки, 48:6 (1990), 91-97.

[7] В. Г. Дринфельд, В. В. Соколов, Докл. АН СССР, 284:1 (1985), 29-33.

[8] A. B. Shabat, R. I. Yamilov, Phys. Lett. A, 130:4-5 (1988), 271-275; А. Б. Шабат, Р. И. Ямилов, Алгебра и анализ, 2:2 (1990), 183-208; А.П. Веселов, А.Б. Шабат, Функи. анализ и его прил., 27:2 (1993), 1-21; A. B. Shabat, R. I. Yamilov, Phys. Lett. A, 227:1-2 (1997), 15-23.

[9] А. Б. Борисов, С. А. Зыков, ТМФ, 115:2 (1998), 199-215.

[10] П. Олвер, Приложения групп Ли к дифференииалъным уравнениям, Мир, М., 1989; Н.Х. Ибрагимов, Применение групп преобразований в математической физике, Наука, М., 1983; А.В. Михайлов, А.Б. Шабат, В.В. Соколов, "Симметрийный подход к классификации интегрируемых уравнений”, Интегрируемость и кинетические уравнения для солитонов, ред. В. Г. Бахтарьяр, В. Е. Захаров, В. М. Черноусенко, Наукова думка, Киев, 1990, 213-279.

[11] A. Sergyeyev, "On recursion operators and nonlocal symmetries of evolution equations", Proc. Sem. Diff. Geom., Math. Publ., 2, ed. D. Krupka, Silesian Univ. Opava, Opava, 2000, 159-173.

[12] В. Г. Дринфельд, В. В. Соколов, “Алгебры Ли и уравнения типа Кортевега-де Фриза”, Итоги науки и техники. Современные проблемы математики, 24, ред. Р. В. Гамкрелидзе, ВИНИТИ, М., 1984, 81-180.

[13] A. G. Meshkov, Nonlocal symmetries of integrable two-field divergent evolutionary systems, arXiv: 0803.1425 .

Поступила в редакцию 9.05.2007, после доработки 7.11.2007 